\title{
Comparison of Lateral Abdominal Muscle Thickness and Cross-Sectional Area of Multifidus in Adults with and without Scoliosis
}

Nahid Rahmani ( $\nabla$ nahrah2005@yahoo.com)

University of Social Welfare and Rehabilitation Sciences

Research article

Keywords: abdominal muscle, multifidus, sonography, muscle thickness, scoliosis

Posted Date: December 12th, 2019

DOl: https://doi.org/10.21203/rs.2.18602/v1

License: (c) (1) This work is licensed under a Creative Commons Attribution 4.0 International License.

Read Full License 


\section{Abstract}

Background: Scoliosis is common in students with a prevalence of $1-2 \%$ in teenagers and more than $50 \%$ in adults $\geq 60$ years. The aim was to compare the abdominal and multifidus muscles size in adults with and without scoliosis.

Methods: Forty men with and without scoliosis were recruited. The Visual Analogue Scale and the Oswestry Disability Questionnaire were used to evaluate the pain intensity and functional disability in the patients' group, respectively. Subjects were asked to lie down in a supine position with their knees bent to measure abdominal muscles using sonography. The linear transducer was placed vertically on the anterolateral abdominal wall to record images of the abdominal muscles (Transversus abdominis, internal oblique, external oblique). To assess the lumbar multifidus muscle size, the curvilinear head was used horizontally on the multifidus muscle at the L5-S1 level in a prone lying position.

Results: No significant difference was found between the two groups for demographic variables. A significant difference was found between the healthy subjects and patients with scoliosis for right and left abdominal and multifidus muscles size $(\mathrm{p}<0.05)$. No significant difference was reported between the two sides of the convexity and concavity on muscles size in patients with scoliosis $(p>0.05)$.

Conclusions: According to the results, patients with scoliosis had smaller abdominal and lumbar multifidus muscles size, but no significant differences were found between the muscle size of the concave and convex sides in the scoliosis group. Future work is needed to support the findings of the current study.

\section{Background}

Scoliosis is a three-dimensional impairment of the vertebral column with deviations in the sagittal and frontal planes, which is usually coupled with vertebral rotation ${ }^{(1)}$. This presents during the childhood or adolescent and could be accompanied by congenital deformity of one or more vertebrae, fracture or dislocation of the vertebrae, lower limb length discrepancy, and poor posture (due to pain or muscular spasm) ${ }^{(2)}$. Scoliosis is common in students ${ }^{(3)}$ with a prevalence of $1-2 \%$ in teenagers and more than $50 \%$ in adults $\geq 60$ years ${ }^{(4)}$. Despite previous reports regarding equal incidence of scoliosis in female and male children, the prevalence seems to rise in females with age, as by the age of 10 the ratio of female to male (with scoliosis) is 6 to 1 , respectively ${ }^{(5)}$.

Trunk muscle imbalance caused by impairment leads to contracture of the muscles on the concave side and stretch on the convex side. In other terms, it may cause asymmetry in the vertebral column muscles ${ }^{(6)}$, which in turn results in lateral deviation and kyphosis ${ }^{(7)}$. Previous studies demonstrated changes in the abdominal muscles and bilateral erector spine activation pattern in patients with scoliosis. In the convex side muscle activation increases, resulting in an increase in the thoracic erector spine muscle in all directions ${ }^{(7,8)}$. The asymmetric forces inserted on the vertebral column (e.g. in gymnastics and ballet 
dancers) coupled with loose ligamentous may disturb vertebral balance mechanism and consequently, incident and progression of scoliosis ${ }^{(9)}$. In patients with scoliosis cross sectional view of muscle fibers reported to be thicker in the concave side compared to the convex side, in the thoracic as well as the thoraco-lumbar, and the lumbar regions ${ }^{(10)}$. The above-mentioned findings need more support through further investigation on the role of muscle function in pathogenesis of scoliosis. To date, several methods have been used to assess the vertebral muscles such as Electromyography $(E M G)(11,12)$, Magnetic resonance imaging (MRI) ${ }^{(13)}$, and Sonography ${ }^{(14-16)}$. Among these tools, sonography has been established as a repeatable, available, non-expensive, and non-invasive tool, with live images which could be used to measure the cross section, antero-posterior, and lateral dimensions, as well as the shape of the muscle ${ }^{(17)}$. Measurement of the muscular dimensions is a valuable index to assess muscle's characteristics ${ }^{(18)}$.

Evidences support that vertebral muscle stabilizers play an important role in dynamic and segmental control of the vertebrae. Therefore, weakness, atrophy, and functional impairments of these muscles could result in vertebral instability. Additionally, asymmetries in the stabilizing muscles size may lead or exaggerate spinal deviations and finally scoliosis. Considering the high prevalence of this impairment and its consequences and the lack of enough evidence on the measures and dimensions of the abdominal and multifidus muscles in this group of patients. The current study was designed to investigate the size of abdominal (transverse abdominis $=\operatorname{TrA}$, internal oblique $=10$ and external oblique $=$ EO) and lumbar multifidus muscle in patients with scoliosis compared to the healthy controls, using ultrasonography.

\section{Methods}

Forty men with and without scoliosis (20 healthy and 20 patients) were recruited for the purpose of this study. All subjects were informed about the aims and the procedures of the study and signed informed consent form if they were willing to take part in the study. Ethical approval was received from the ethical committee of University of Social Welfare and Rehabilitation.

Patients with scoliosis were men between ages of $20-50$, clinically and radiographically diagnosed with scoliosis and low back pain due to scoliosis by a spine surgeon and were referred to the USWR outpatient clinic. Healthy controls were selected from men between $20-50$ years, without any history of low back pain and scoliosis. The general exclusion criteria for all groups were a history of sacroiliac joint dysfunction, respiratory/rheumatologic/neurologic disorders, fracture/dislocation of the spine, metabolic diseases, spondylolysis/spondylolisthesis, and being allergic to sonography gel. The above-mentioned information was collected subjectively through a self-administered questionnaire.

In the patients' group the Visual Analogue Scale (VAS) ${ }^{(19)}$ and the Oswestry Disability Questionnaire $(\mathrm{ODQ}){ }^{(20)}$ were used to assess the pain intensity and functional disability, respectively. 
The portable ultrasonic diagnosis equipment, model LEO-3000D1 (XuZhou LEO Medical Equipment, Co., Ltd) was applied to measure the thickness of abdominal and multifidi muscles. For measurement of antero-lateral abdominal wall's muscle thickness, subjects were asked to lie down in a supine position with knees bent. The sonography transducer (linear head) was placed vertically on the anterolateral abdominal wall, midway between the $12^{\text {th }}$ rib and the anterior superior iliac crest $(21,22)$. Images were recorded, when a clear picture of all three lateral abdominal muscles (TrA, IO and EO)) was obtained (Figure 1).

To assess the lumbar multifidus muscle, participants were positioned in prone lying, with a pillow placed under the abdomen to minimize the lumbar lordosis. Detection of spinous processes (L5-S1) was determined manually using the iliac crests as a landmark. The curvilinear head was used horizontally on the multifidus muscle at the L5-S1 level, which is proved to have the best view ${ }^{(23)}$. The image of the multifidus muscle thickness is shown in Figure 2.

\section{Statistical analysis}

Statistical analysis was performed using SPSS software (version 20, Chicago: SPSS Inc). Descriptive statistics were used to report demographic and clinical characteristics of subjects. Shapiro-Wilk test was used to assess the normal distribution of desired variables. An independent t-test was applied to evaluate possible differences between the two groups. To assess the relationship between variables, a Pearson Correlation Coefficient was employed. The level of significance was set at $P<0.05$.

\section{Results}

Demographic characteristics and muscle thickness were provided in Table 1 . Using Shapiro-Wilk test, the two groups were homogenous at baseline for demographic variables and the distribution of variables was normal.

Mean and standard deviation of demographic variables and abdominal and multifidus muscles size in healthy and patient groups are shown in Table 1. 
Table 1. Mean and standard deviation of demographic variables and muscles sizes in healthy and patient groups

\begin{tabular}{|c|c|c|c|c|}
\hline \multicolumn{2}{|c|}{ Patient } & \multicolumn{2}{|c|}{ Healthy } & \multirow[t]{2}{*}{ Variables } \\
\hline Range & Mean (SD) & Range & Mean (SD) & \\
\hline \multicolumn{5}{|c|}{ Demographic variables } \\
\hline $20-50$ & $35.3(9.1)$ & $21-49$ & $33.7(8.2)$ & Age (year) \\
\hline $160-180$ & $170.7(5.1)$ & $182-164$ & $172.3(5.0)$ & Height (cm) \\
\hline $55-87$ & $69.8(7.7)$ & $60-95$ & $74.0(9.3)$ & Weight (kg) \\
\hline $20.5-31.8$ & $24.2(2.5)$ & $21.2-30.7$ & $23.0(2.8)$ & BMI $(\mathrm{kg} / \mathrm{m} 2)$ \\
\hline 10.28 & $16.3(2.5)$ & $\mathrm{N} / \mathrm{A}$ & $\mathrm{N} / \mathrm{A}$ & Deviation degree () \\
\hline \multicolumn{5}{|c|}{ Muscles size (cm) } \\
\hline $0.30-0.46$ & $0.46(0.11)$ & $0.48-0.80$ & $0.61(0.09)$ & RtTrA \\
\hline $0.30-0.73$ & $0.47(0.10)$ & $0.45-0.73$ & $0.60(0.08)$ & LtTrA \\
\hline $0.86-1.23$ & $0.86(0.16)$ & $0.73-1.97$ & $1.30(0.35)$ & RtIO \\
\hline $0.57-1.26$ & $0.85(0.16)$ & $0.78-1.94$ & $1.31(0.32)$ & LtIO \\
\hline $0.55-0.75$ & $0.55(0.09)$ & $0.55-1.13$ & $0.75(0.15)$ & RtEO \\
\hline $0.42-0.78$ & $0.55(0.09)$ & $0.50-0.91$ & $0.74(0.10)$ & LtEO \\
\hline $0.92-1.90$ & $1.63(0.19)$ & $1.50-2.77$ & $2.01(0.31)$ & $\operatorname{RtMF}\left(\mathrm{cm}^{2}\right)$ \\
\hline $0.93-1.90$ & $1.64(0.19)$ & $1.54-2.01$ & $1.97(0.26)$ & $\operatorname{LtMF}\left(\mathrm{cm}^{2}\right)$ \\
\hline
\end{tabular}

RtTrA=Right Transversus Abdominis; LtTrA=Left Transversus Abdomini; RtIO=Right Internal Oblique; LtIO=Left Internal Oblique; RtEO=Right External Oblique; LtEO=Left External Oblique; RtMF=Right Multifidus; LtMF=Left Multifidus

In Table 2, the results of an independent t-test to assess the probable difference between healthy and patient groups on abdominal and multifidus muscle size were demonstrated. The results (Table 3) indicated a significant difference between healthy subjects and those suffering from scoliosis in terms of right and left abdominal and multifidus muscles size ( $\mathrm{p}<0.05$ in all instances).

Table 2. Mean difference of the abdominal and multifidus muscles size between healthy and patient groups $(\mathrm{n}=20)$ 


\begin{tabular}{|l|l|l|l}
\hline P-value & t-value & Mean difference & Variables (cm) \\
\hline 0.02 & 2.24 & 0.14 & RtTrA \\
\hline 0.03 & 2.16 & 0.12 & LtTrA \\
\hline 0.00 & 5.01 & 0.43 & RtIO \\
\hline 0.01 & 5.70 & 0.46 & LtIO \\
\hline 0.01 & 3.93 & 0.20 & RtEO \\
\hline 0.02 & 3.15 & 0.18 & LtEO \\
\hline 0.02 & 4.65 & 0.38 & RtMF (cm $\left.{ }^{2}\right)$ \\
\hline 0.02 & 4.52 & 0.33 & LtMF (cm $\left.{ }^{2}\right)$ \\
\hline
\end{tabular}

RtTrA=Right Transversus Abdominis; LtTrA=Left Transversus Abdomini; RtIO=Right Internal Oblique; LtIO=Left Internal Oblique; RtEO=Right External Oblique; LtEO=Left External Oblique; RtMF=Right Multifidus; LtMF=Left Multifidus

Possible differences on the abdominal and multifidus muscles size between the convexity and concavity sides in patients with scoliosis using paired t-test are shown in Table 3. The results (Table 3) demonstrated no significant difference between the two sides of the convexity and concavity on muscles size in patients with scoliosis ( $p>0.05$ in all instances).

Table 3. Mean difference of the abdominal and multifidus muscles size between convexity and concavity sides in patient with scoliosis $(\mathrm{n}=20)$

\begin{tabular}{|l|l|l|l}
\hline P-value & t-value & Mean difference & Variables (cm) \\
\hline 0.61 & 0.51 & 0.01 & TrA \\
\hline 0.20 & 1.30 & 0.03 & IO \\
\hline 0.37 & 0.90 & 0.01 & EO \\
\hline 0.39 & 0.87 & 0.01 & MF $\left(\mathrm{cm}^{2}\right)$ \\
\hline
\end{tabular}

TrA=Transversus Abdominis; IO=Internal Oblique; EO=External Oblique; MF=Multifidus 
The aim of this study was to compare the lateral abdominal muscle thickness and cross-sectional area of multifidus between individuals with and without scoliosis. Previous research demonstrated that abdominal and multifidus muscle thickness is decreased in patients with low back pain compared to their healthy peers. In a study conducted by Zapata et al, deep paraspinal muscle thickness (T8, L1, and L4 levels) were compared between teenagers with and without idiopathic scoliosis ${ }^{(24)}$. Results showed that muscle thickness in all three levels were higher at the concave side in subjects with scoliosis, compared to the healthy subjects, which is in line with the findings in the current study ${ }^{(24)}$. They also reported an increased muscle thickness at the concave side of patients with scoliosis compared to the convex side in both T8 and L1 levels ${ }^{(24)}$. This finding was not consistent with the results of the current study and this difference might be might be attributed to some methodological differences between the two studies such as different sample size, age difference between participants of the two studies, as well as different multifidus levels of assessment. In the current study the L5-S1 level was studied and, in the study conducted by Zapata et al, the L1 and L4 levels were investigated.

Kim et al, assessed the activity of the transverse abdominus muscle in maximum exhale, and compared the results between patients with chronic low back pain and healthy controls ${ }^{(25)}$. Results demonstrated that although transverse abdominus muscle thickness was different in both resting and contraction in patients with chronic back pain compared to the controls, muscle activation changes were not significantly different. Also, the level of atrophy of the transverse abdominus muscle in patients with chronic back pain was variable. Observed differences in muscle thickness and activation of transverse abdominus in this group of patients might be due to possible changes in movement pattern and not necessarily in the excitability of motor neurons ${ }^{(25)}$. Pain can affect muscle activation and may result in movement control impairments. Therefore, the morphologic changes in transverse abdominus muscle, might be responsible for the beginning of movement control impairments and subsequent back pain ${ }^{(25)}$.

In a study by Whittaker et al, carried out on patients with and without Lumbo-pelvic pain, abdominal muscle (TrA, IO, EO, and rectus abdominus) thickness and surrounding soft tissue were assessed using ultrasonography. Only the rectus abdominus muscle thickness was shown to be significantly less in patients with lumbo-pelvic pain compared to the healthy controls ${ }^{(26)}$. This finding was not consistent with current results, which might be due to the fact that patients experienced low back pain (due to scoliosis) in this study compared with acute low back pain of those in Whitaker et al, study.

Wallwork et al, investigated lumbar multifidus muscle size during contraction, as well as the ability of the muscle for maximum voluntary isometric contraction, using ultrasonography in subjects with and without back pain ${ }^{(27)}$. They reported a significantly smaller cross section area for multifidus muscles at L5 level in patients with back pain compared to the healthy controls. They also reported that the percentage of muscle thickness during contraction was significantly less in low back pain patients compared to the healthy controls ${ }^{(27)}$. Similarly, Hides et al, reported significant decrease in cross sectional view of the multifidus muscle at the L4-L5 level in patients with back pain compared to the healthy controls, which 
was accompanied by an increase in the maximum asymmetry at $L 5$ level in patients with unilateral pain (28).

Previous findings demonstrated that abdominal and lumbar multifidus muscle thickness as well as muscle activity is decreased in patients with low back pain and those with scoliosis compared with healthy subjects.

\section{Conclusions}

In conclusion, results from the current study indicate that the thickness of abdominal muscles (including the transverse abdominis, internal oblique and external oblique) and cross-sectional area of lumbar multifidus were smaller in patients with scoliosis compared with healthy subjects. No significant differences were found between the muscle thickness in the concave and convex sides in the patients with scoliosis. However, further study is needed to support the findings of the current study.

\section{Abbreviations}

EMG:electromyography; MRI:magnetic resonance imaging; TrA:transverse abdominis; IO:internal oblique; EO:external oblique; USWR:university of social welfare and rehabilitation; VAS:visual analogue scale; ODQ:oswestry disability questionnaire; RtTrA:right transversus abdominis; LtTrA:left transversus abdominis; RtIO:right internal oblique; LtIO:left internal oblique; RtEO:right external oblique; LtEO:left external oblique; RtMF:right multifidus; LtMF:left multifidus; MF:multifidus; SD:standard deviation.

\section{Declarations}

Ethics approval and consent to participate: This project received approval from the ethical committee of the university of social welfare and rehabilitation sciences and all participants signed the consent form of the study.

The full name of ethical committee: the ethical committee of University of Social Welfare and Rehabilitation sciences.

Consent for publication: All authors reviewed the manuscript and gave their consent for publication.

Availability of data and material: The data are available for the research team.

Competing interests: There is no competing interests in this study.

Funding: The funding was provided by the university of social welfare and rehabilitation sciences.

Authors' contributions: All authors $\left(\mathrm{MAMB}^{1}, \mathrm{AK}^{2}, \mathrm{NR}^{3}, \mathrm{SAB}^{4}, \mathrm{AM}^{5}\right)$ contributed in the different sections of the study. 
Acknowledgements: We acknowledge the patients participated in the study and the university of social welfare and rehabilitation sciences for supporting this project.

\section{References}

1. Meier MP, Klein MP, Krebs D, Grob D, Muntener M: Fiber transformations in multifidus muscle of young patients with idiopathic scoliosis. Spine. 1997;22(20):2357-2364.

2. Salter RB: Textbook of disorders and injuries of the musculoskeletal system. second edition. Williams \& Wilkins, Baltimore, Md; 1983.

3. Willner S, Uden A. A prospective prevalence study of scoliosis in southern Sweden. Acta Orthopaedica Scandinavica. 1982; 53(2): 233-237.

4. Carter OD, Haynes S. Prevalence rates for scoliosis in US adults: results from the first national health and nutrition examination survey. International Journal of Epidemiology. 1987; 16(4): 537-544.

5. Trobisch P, Suess O, Schwab F. Idiopathic scoliosis. Deutsches Arzteblatt International. 2010;107(49):875-83.

6. Kisner C, Brody LA. Exercicios terapeuticos. Fundamentos e tecnicas. Sao Paulo: Manole; 1998.

7. Cheung J, Veldhuizen AG, Halberts JP, Sluiter WJ, and Van Horn JR. “Geometric and electromypgraphic assessments in the evaluation of curve progression in idiopathic scoliosis. Spine. 2006; 31(3): 322-329.

8. Odermatt D, Mathieu PA, Bequsejour M, Labelle $H$, and Aubin CE. Electromyography of scoliotic patients treated with a brace. Journal of Orthopaedic Research. 2003; 21(5): 931-936.

9. Tanchev PI, Dzherov AD, Parushev AD, Dikov DM, Todorov MB. Scoliosis in rhythmic gymnasts. Spine. 2000;25(11):1367-1372.

10. Mannion AF, Dumas GA, Cooper RG, Espinosa FJ, Faris MW, Stevenson JM. Muscle fiber size and type distribution in thoracic and lumbar regions of erector spinae in healthy subjects without low back pain: normal values and sex differences. Journal of anatomy. 1997;190(4):505-513.

11. McMeeken JM, Beith ID, Newham DJ, Milligan P, Critchley DJ. The relationship between EMG and change in thickness of transversus abdominis. Clinical Biomechanics. 2004; 19(4): 337-342.

12. Mohseni-Bandpei MA, Critchley J, Staunton T, Richardson B. Application of surface electromyography in the assessment of low back pain: A Literature Review Physical Therapy Reviews. 2000;5(2):93-105.

13. Hides $\mathrm{J}$, Wilson $\mathrm{S}$, Stanton W. An MRI investigation into the function of the transversus abdominis muscle during "drawing-in" of the abdominal wall. Spine. 2006; 31(6): E175-E178.

14. Nabavi N, Mosallanezhad Z, Haghighatkhah HR, Bandpeid MAM. Reliability of rehabilitative ultrasonography to measure transverse abdominis and multifidus muscle dimensions. Iranian Journal of Radiology. 2014;11(3):1-4.

15. Rahmani N, Mohseni-Bandpei MA, Vameghi R, Salavati M, Abdollahi I. Application of ultrasonography in the assessment of skeletal muscles in children with and without neuromuscular 
disorders: A systematic review. ultrasound in Medicine \& Biology. 2015;41(9):1-9.

16. Rahmani N, Mohseni-Bandpei MA, Vameghi R, Salavati M, Abdollahi I. Normal values of abdominal muscles thickness in healthy children using sonography. Musculoskeletal Science and Practice. 2018; 34: 54-58.

17. Whittaker JL, Teyhen DS, Elliott JM. Rehabilitative ultrasound imaging: understanding the technology and its applications. The Journal of Orthopaedic and Sports Physical Therapy. 2007; 37(8): 434-449.

18. Rankin G, Stokes M, Newham DJ. Size and shape of the posterior neck muscles measured by ultrasound imaging: normal values in males and females of different ages. Manual Therapy.2005; 10(2): 108-115.

19. Waterfield J, Sim J. Clinical assessment of pain by visual analogue scale. British Journal of Therapy and Rehabilitation. 1996; 3(2): 94-97.

20. Fairbank JCT, Pynsent PB. The Oswestry disability index. Spine. 2000;25(22):2940-2953.

21. Pulkovski N, Mannion AF, Caporaso F, et al. Ultrasound assessment of transversus abdominis muscle contraction ratio during abdominal hollowing: a useful tool to distinguish between patients with chronic low back pain and healthy controls? European Spine Journal. 2012; 21(6): S750-S759.

22. Hides JA, Miokovic T, Belavy DL, Stanton WR, Richardson CA. Ultrasound imaging assessment of abdominal muscle function during drawing-in of the abdominal wall: an intrarater reliability study. Journal of Orthopaedic and Sports Physical Therapy. 2007;37(8):480- 486.

23. Hides JA SW, McMahon S, Sims K, Richardson CA. Effect of stabilization training on multifidus muscle cross-sectional area amongyoung elite cricketers with low back pain. Journal of Orthopaedic Sports Physical Therapy. 2008;38(3):101-8.

24. Zapata KA, Wang-Price SS, Sucato DJ, Dempsey-Robertson M. Ultrasonographic measurements of paraspinal muscle thickness in adolescent idiopathic scoliosis: A comparison and reliability study. Pediatric Physical Therapy. 2015; 27(2): 119-25.

25. Kim KH CS, Goo BO, Baek IH. Differences in transversus abdominis muscle function between chronic low back pain patients and healthy subjects at maximum expiration: Measurement with Real-time Ultrasonography. Journal of Physical Therapy Science. 2013;25(7):861-3.

26. Whittaker JL, Warner MB, Stokes M. Comparison of the sonographic features of the abdominal wall muscles and connective tissues in individuals with and without lumbopelvic pain. Journal of Orthopaedic and Sports Physical Therapy. 2013; 43(1): 11-19.

27. Wallwork TL, Stanton WR, Freke M, Hide J. The effect of chronic low back pain on size and contraction of the lumbar multifidus muscle. Manual Therapy. 2009; 14(5): 496-500.

28. Hides J, Gilmore C, Stanton WR, Bohlscheid E. Multifidus size and symmetry among chronic LBP and healthyasymptomatic subjects. Manual Therapy. 2008; 13(1): 43-49.

\section{Figures}




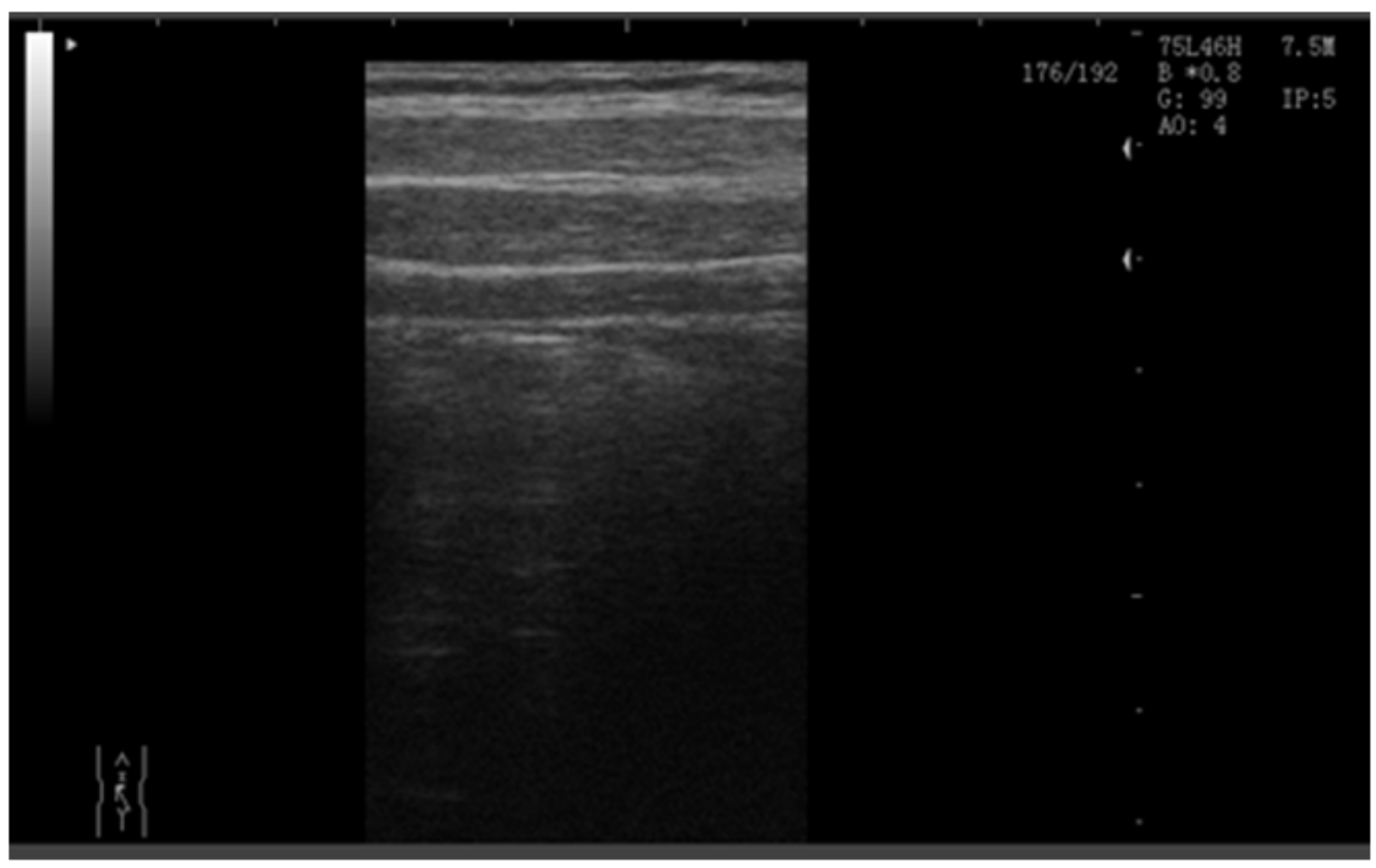

Figure 1

The image of the abdominal muscles thickness 


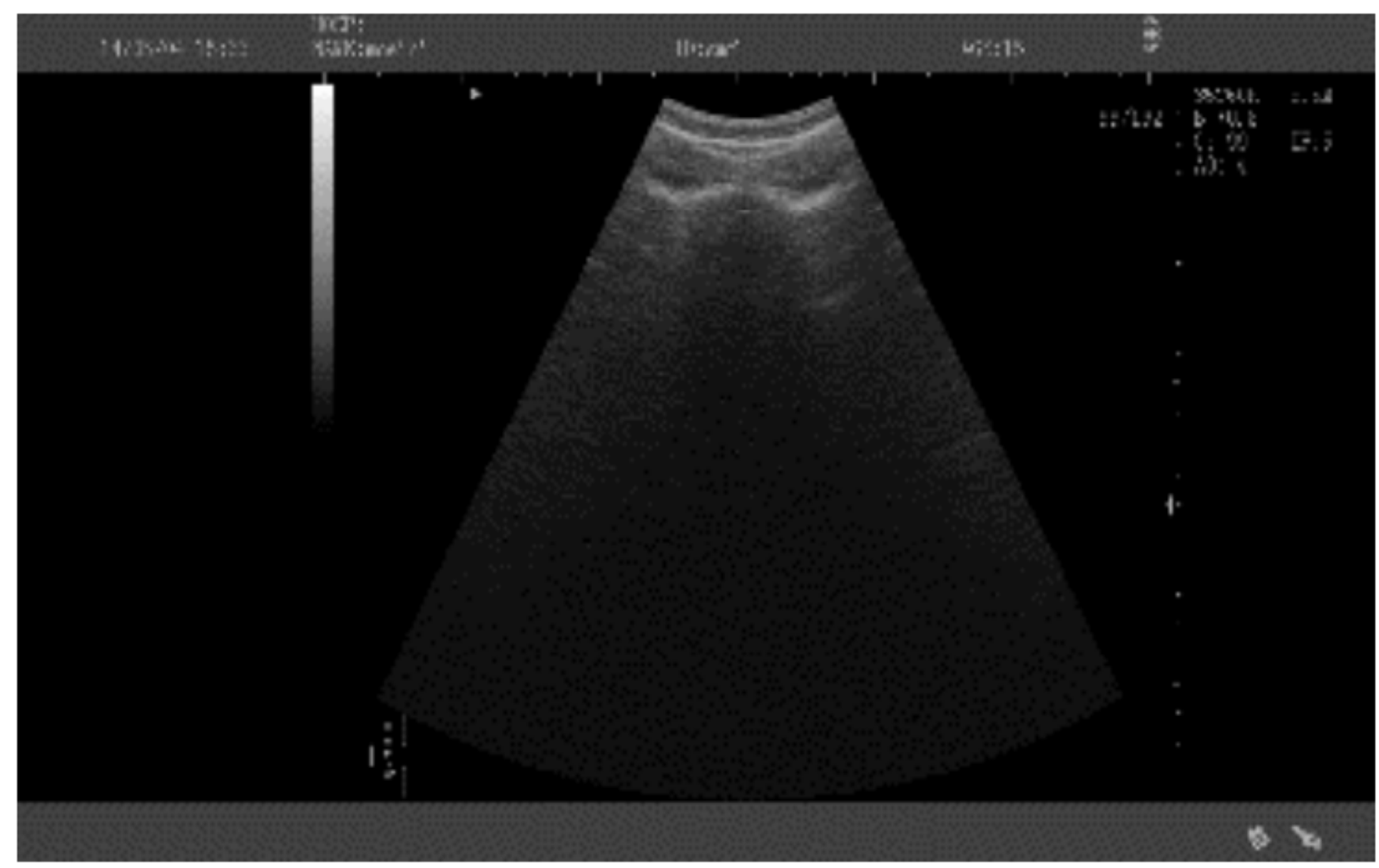

Figure 2

The image of the multifidus muscle thickness 